\title{
Erythema Marginatum In A Case Of Post-Streptococcal Arthralgia: A Rash Mimicking Urticaria
}

\author{
G Wilson, J Dyer, D Folzenlogen
}

\begin{abstract}
Citation
G Wilson, J Dyer, D Folzenlogen. Erythema Marginatum In A Case Of Post-Streptococcal Arthralgia: A Rash Mimicking Urticaria. The Internet Journal of Rheumatology. 2010 Volume 7 Number 1.
\end{abstract}

DOI: $\underline{10.5580 / \mathrm{e} 4 \mathrm{~d}}$

\begin{abstract}
Erythema marginatum secondary to streptococcal infection is classically associated with acute rheumatic fever and fulfills one of the Jones criteria for this diagnosis. We describe a case of streptococcal associated erythema marginatum without rheumatic heart disease in a 12 year old male with post streptococcal arthralgia and myalgia. The rash was originally suggestive of urticaria. He was initially seen with a five week history of fever. Examination and laboratory evaluation did not yield an acute infectious etiology for his fever. However anti-streptolysin $\mathrm{O}$ and anti-deoxyribonuclease $\mathrm{B}$ were very elevated. Historically, he had a sore throat 3 weeks prior to his presentation and had been treated by his PCP with amoxicillin; no cultures were done. Based on this history and his elevated anti-streptococcal antibody titers, he was started on cefadroxil and non-steroidal antiinflammatory drugs. Several days after antibiotics were started and several weeks after his fever and arthralgia symptoms had begun, he developed a trunk and upper arm rash with raised, erythematous borders. Dermatology diagnosed it as urticaria and cetirizine was recommended. The antibiotic was switched to azithromycin but the rash persisted. He was referred to rheumatology who diagnosed a post-streptococcal syndrome. He was started on penicillin under close observation and no changes in the rash were noted. His aches improved in a matter of days. Dermatology biopsied his rash and the histopathology was consistent with both urticaria and published reports of erythema marginatum. Electrocardiogram and serial echocardiographs were normal. His anti-streptococcal titers returned to near normal in 12 months. His elevated inflammatory markers normalized. This case demonstrates a post-streptococcal syndrome with a rash suggestive of urticaria, but was later confirmed to be erythema marginatum in the absence of carditis. Confirming the diagnosis of erythema marginatum was critical, since it enabled a diagnosis of rheumatic fever which had direct implications for prophylactic treatment.
\end{abstract}

\section{INTRODUCTION}

Post-streptococcal syndromes, such as rheumatic fever and post-streptococcal arthritis, have diagnostic criteria. Many post-streptococcal illnesses may not fulfill diagnostic requirements. The breadth of post-streptococcal syndromes is increasingly apparent. Diagnosing a post-streptococcal syndrome is often difficult, not only in meeting criteria but also making a temporal association with streptococcal exposure. The syndromes may overlap in presentation, may be incomplete, or may mimic other systemic disease. We present a case of temporally related post-streptococcal arthralgia and myalgia with erythema marginatium without evidence of carditis. The rash was initially diagnosed as urticaria.

\section{CASE PRESENTATION}

A 12 year old male was admitted with no significant past medical history. He first presented to the infectious disease clinic with a five week history of fevers to $103 \mathrm{~F}$ and a 3 week history of polyarthralgia and myalgia. The joint discomfort was limited to his shoulders, knees, ankles and his mandible area. The discomfort caused him to miss several weeks of school. The pain partially responded to non-steroidal anti-inflammatory drugs (NSAIDs). His review of systems revealed a history of sore throat 3 weeks prior to presentation, which had been treated with amoxicillin. His exam revealed an antalgic gait but no erythema or joint swelling. In addition, no limitation of joint movement was seen. He had no muscle tenderness or weakness on exam. His cardiac, pulmonary, lymphatic and abdominal exams were normal.

His workup included an elevated erythrocyte sedimentation rate of $74 \mathrm{~mm} / \mathrm{hr}(0-15 \mathrm{~mm} / \mathrm{hr})$, a c-reactive protein of 8.9 $\mathrm{mg} / \mathrm{dL}(<=0.5 \mathrm{mg} / \mathrm{dL})$, mild anemia with hemoglobin of $11.5 \mathrm{~g} / \mathrm{dL}$ (12.5-16.1 g/dL), white blood count of 6700 
(4000-12,000) and increased platelets at 587,000 (150-400 $10-3 / \mathrm{mcL}$ ). Computed tomography of his mandible, ultrasound of his abdomen, and radiograph of his chest were normal. Blood cultures were negative and urinalysis initially showed trace protein which was not seen on subsequent samples. Creatinine phosphokinase levels and serum chemistries were normal with the exception of an elevated total protein of $9.0 \mathrm{~g} / \mathrm{dL}$. Evaluation for Ehrlichia, Rocky Mountain Spotted Fever, Bartonella, cytomegalovirus, and Epstein-Barr virus were normal, but his anti-streptolysin $\mathrm{O}$ (ASO) was elevated at $2490 \mathrm{IU} / \mathrm{mL}$ (normal 0-240 IU/mL) and his anti-deoxyribonuclease B (anti-DNase B) was increased to 797 units/mL (normal 0-170 units/mL). Hepatitis B and C serologies were also normal. Echocardiography showed no valvular disease and an electrocardiogram (ECG) showed a normal P-R interval. The available data and exam findings supported a streptococcal related illness and infectious disease placed the patient on cefadroxil.

Several weeks after his fever and sore throat began and within 3 days of starting cefadroxil, he developed a serpiginous, raised rash with erythematous borders on his torso and upper arm (Figure 1).

\section{Figure 2}

Figure 1

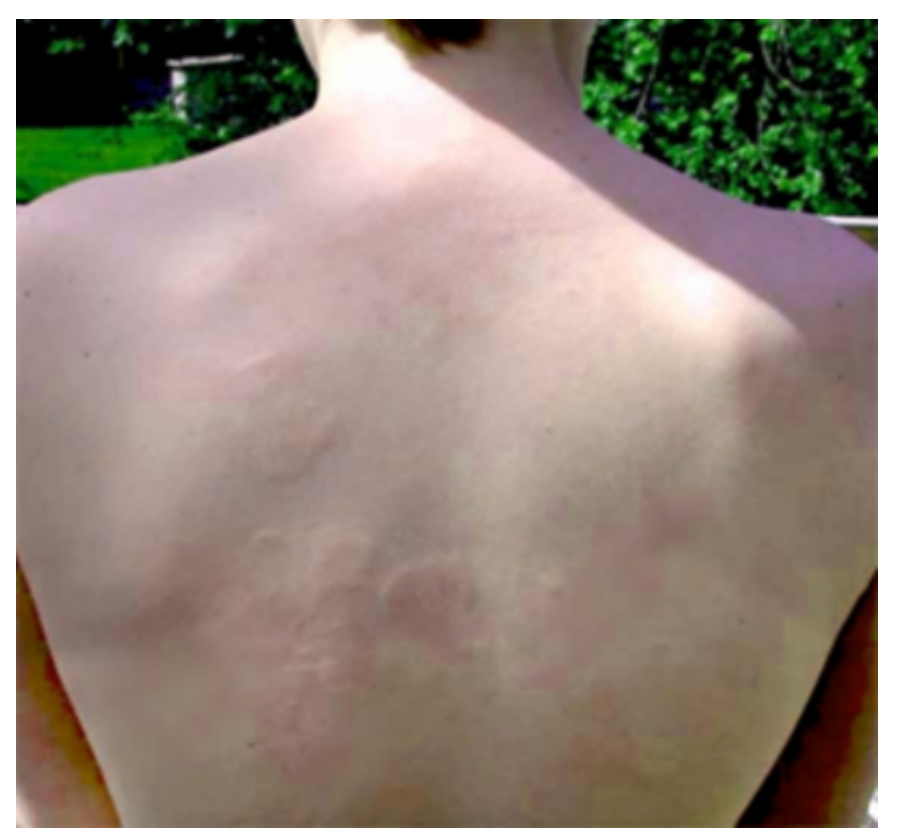

His antibiotic was changed to azithromycin but the rash continued. He was referred to dermatology and rheumatology. Dermatology diagnosed the rash as having urticarial characteristics but they did not think it was secondary to the antibiotics. He was prescribed cetirizine.
The patient's mother felt the rash was unchanged with the cetirizine; therefore, he did not take it regularly.

Rheumatology also evaluated the patient, reviewed the previous laboratory and diagnostic data, and concluded that the patient had a post-streptococcal syndrome. Under close observation, penicillin was started.

At a subsequent dermatology appointment, the patient's rash was biopsied and showed "... an interstitial admixture of neutrophils and eosinophils and dermal edema. The findings are not entirely diagnostic of urticaria, as the amount of inflammation is slightly more than typically seen in urticaria..." $\left(\mathrm{RFC}^{\mathrm{c}}\right)$. The rash appeared to be consistent with erythema marginatum (EM) based on the clinical findings and the published biopsy reports. The intensity and morphology of the lesions varied over time, but they consistently had a red/pink hue with raised edges (Figures 1 and 2). After following the patient for several months, dermatology re-evaluated the rash (figure 2) and agreed that it was consistent with EM.

\{image: 2$\}$

The patient's arthralgia decreased within one week of initiating penicillin, but the jaw symptoms and upper arm myalgia continued to be intermittent for several months before stopping. In addition over a nine month period, his inflammatory markers normalized, ASO and anti-DNase B titers decreased to near normal, and the total protein decreased to normal (corresponding with the decline in ASO and anti-DNase B titers). Clinically, he has returned to his baseline and has no arthralgia or myalgia. His rash diminished after nine months of antibiotics, but is still faintly present on is back (Figure 2). Based on the diagnosis of EM, the patient meets criteria for acute rheumatic fever without carditis. In this case, it is recommended he be treated prophylactically for 5 years.

\section{DISCUSSION}

Post-streptococcal syndromes, including acute rheumatic fever, post-streptococcal reactive arthritis, post-streptococcal related myalgia/myositis, and post-streptococcal glomerulonephritis, manifest in multiple organ systems and do not always clearly fulfill defined diagnostic criteria.

Meeting criteria and confirming streptococcal disease (exposure) creates a diagnostic challenge for the clinician.

Classically, post-streptococcal syndromes occur 1-3 weeks after pharyngitis or skin infection with peak antistreptococcal antibodies seen in 3-8 weeks ${ }^{1,2}$, although antibody titers may persist for months. In some patients, 
antibody titers never increase. This may be due to infections caused by different Lancefield groups or emm genotype ${ }^{3}$.

Several immune mediated mechanisms for developing streptococcal related illness have been postulated, including antibody directed molecular mimicry in rheumatic fever, immune complex deposition in post-streptococcal glomerulonephritis and possible super antigen stimulation of T cells in Kawasaki disease and guttate psoriasis ${ }^{4-6}$.

Increased awareness of the role of streptococcus in these diseases is essential as the estimated worldwide prevalence of severe group A streptococcal disease (e.g. acute rheumatic fever, rheumatic heart disease, post-streptococcal glomerulonephritis, and invasive infection) is 18 million cases annually ${ }^{7}$.

EM was confirmed in our patient after serial examinations in addition to a skin biopsy which showed a combination of interstitial neutrophils, eosinophils and dermal edema. The histopathology of EM was initially described in 1935 by Carol and van Krieken who described it as a perivascular infiltrate in the upper dermis composed predominantly of neutrophils with a few lymphocytes and eosinophils ${ }^{8}$. Nuclear debris suggesting leukocytoclasia is often seen, although vasculitis is not ${ }^{9,10}$. Our biopsy specimen is consistent with these descriptions of EM. This patient's clinical picture is also consistent with EM as a poststreptococcal syndrome manifestation.

Erythema marginatum is one of the major Jones criteria for acute rheumatic fever ${ }^{11}$. When applied to our patient, these criteria support the diagnosis of acute rheumatic fever as he has one major and four minor criteria. However, the initial ECG and serial echocardiography revealed no carditis or valvular disease. Our case is unique in that our patient's clinical pattern is consistent with acute rheumatic fever without carditis and satisfies the Jones criteria for this diagnosis. Without an accurate diagnosis of EM, the diagnosis of rheumatic fever cannot be made as no major criteria would exist. Multiple cardiac evaluations did not yield evidence for cardiac involvement, but close cardiac monitoring will continue as cases of carditis in ARF have been diagnosed at a year or more after the initial diagnosis. We present this case of an atypical presentation of a poststreptococcal manifestation in order to raise awareness of not only the varying manifestations of these syndromes but also the importance of an accurate dermatological diagnosis.

\section{ACKNOWLEDGEMENTS}

The authors would like to thank Ms. Crystal Arellano for her technical assistance.

\section{References}

1. Blyth, CC and Robertson, PW: Anti-streptococcal antibodies in the diagnosis of acute and post-streptococcal disease: streptokinase versus streptolysin $\mathrm{O}$ and deoxyribonuclease B. Pathology; 2006; Vol 38, N 2: 152-156.

2. Ivory, D and Folzenlogen, D: Post streptococcal syndromes, a rheumatologist perspective. The Internet Journal of Rheumatology; 2010; Vol 6, Number 2.

3. Johnson, DR, Kurlan, R, Leckman, J, and Kaplan, EL:

The Human Immune Response to Streptococcal

Extracellular Antigens: Clinical, Diagnostic, and Potential

Pathogenic Implications. Clinical Infectious Diseases; 2010;

Vol 50, N 5: 481-490.

4. Samarkos, $M$ and Vaiopoulos, G: The role of infections in the pathogenesis of autoimmune diseases. Current Drug

Targets- Inflammation \& Allergy; 2005; Vol 4, N 1: 99-103.

5. Martins, TB, Veasy, LG, and Hill, HR: Antibody responses to group A streptococcal infections in acute rheumatic fever. The Pediatric Infectious Disease Journal; 2006; Vol 25, N 9: 832-837.

6. Davison, SC, Allen, MH, Mallon, E, and Barker, JNWN: Contrasting patterns of streptococcal superantigen-induced $\mathrm{T}$-cell proliferation in guttate vs. chronic plaque psoriasis. British Journal of Dermatology; 2001; Vol 145, N 2: 245-251.

7. Carapetis, JR, Steer, AC, Mulholland, EK and Weber, M:

The global burden of group A streptococcal diseases. Lancet Infectious Diseases; 2005; Vol 5, N 11: 685-694.

8. Carol, W and van Krieken, J: Zur histopathologie des erythema annulare von Lehndorff and Leriner. Acta Paediatrica; 1935; Vol 17, N 3: 372-376.

9. Troyer, C, Grossman, ME, and Silvers, DN: Erythema marginatum in rheumatic fever: Early diagnosis by skin biopsy. Journal of the American Academy of Dermatology; 1983; Vol 8, N 5, 724-728.

10. Sahn, EE, Maize, JC, and Silver, RM: Erythema marginatum: An unusual histopathology manifestation. Journal of the American Academy of Dermatology; 1989; Vol 21, N1: 145-147.

11. Saxena, A: Diagnosis of rheumatic fever: current status of Jones criteria and role of echocardiography. Indian Journal of Pediatrics; 2000; Vol 67, N 4: 283-286. 


\section{Author Information}

\section{Gregory J. Wilson}

Division of Immunology and Rheumatology, University of Missouri

\section{Jonathan A. Dyer}

University Physicians Medical Building

\section{Darcy D. Folzenlogen}

Boyce and Bynum Pathology Professional Services, Inc 